\title{
TRACE FUNCTIONS ON IWAHORI-HECKE ALGEBRAS
}

\author{
MEINOLF GECK \\ UFR de Mathématiques, Université Paris 7 \\ 2 Place Jussieu, F-75251 Paris Cedex 05, France \\ E-mail: geck@math.jussieu.fr
}

\begin{abstract}
This paper is an expanded version of a talk given at the Banach Center Symposium on Knot Theory in July/August 1995. Its aim is to provide a general survey about trace functions on Iwahori-Hecke algebras associated with finite Coxeter groups. The so-called Markov traces are relevant to knot theory as they can be used to construct invariants of oriented knots and links. We present a classification of Markov traces for the classical types $A, B$ and $D$.
\end{abstract}

1. Introduction. The algebras in the title first came up in Iwahori's work (cf. [CR], $\S 67)$ on endomorphism algebras of certain representations of finite Chevalley groups. From this point of view, they are relevant to the representation theory of finite groups. Alternatively, such an algebra can be described as a finite dimensional quotient of the group algebra of an Artin-Tits braid group. In the case where the underlying Coxeter group is a symmetric group, Jones [Jo] used this approach to construct invariants for isotopy classes of oriented knots and links in the 3-sphere. (For generalizations to Coxeter groups of type $B$, see [La].) In this article, we shall regard these algebras from a purely algebraic point of view, as deformations of the group algebras of finite Coxeter groups.

Our aim is to describe a general plan for studying trace functions on Iwahori-Hecke algebras associated with any given type of finite Coxeter group. This plan has been developed in joint work with G. Pfeiffer [GP]. As an application, we present a classification of so-called Markov traces on Iwahori-Hecke algebras of classical type. For type $A$, this is originally due to Ocneanu; for type $B$, this is joint work with S. Lambropoulou [GL]. We shall give a self-contained account of the uniqueness part in the latter result but refer the reader to $[\mathrm{GL}]$ for the technically more complicated details of the existence part. We then show how the classification of Markov traces for type $B$ can be used to classify Markov traces for type $D$. These results are new; they are motivated by discussions with S. Lambropoulou which are gratefully acknowledged.

1991 Mathematics Subject Classification: Primary 57M25; Secondary 20C15.

The author thanks G. Pfeiffer for critical comments on the paper.

The paper is in final form and no version of it will be published elsewhere. 
In Section 2 we define and state the main properties of finite Coxeter groups and describe normal forms of the elements for the classical types $A, B$ and $D$. In Section 3 we study conjugation in these groups by examining the signed cycle type of elements, following Carter's description in $[\mathrm{Ca}]$. The associated Iwahori-Hecke algebras and their trace functions are the subject of Section 4. We show how the operation of conjugation in the Coxeter group translates to the Iwahori-Hecke algebra. This will be the key step in describing our general plan for trace functions, along the lines of [GP]. In Section 5, we introduce Markov traces for Iwahori-Hecke algebras of classical type, and study some of their basic properties. Finally, in Section 6, we give a classification of Markov traces in the case of type $D$. This is followed by some concluding remarks and open questions.

We have tried to keep the level of our exposition as elementary as possible. We assume known the notions of finitely presented groups and associative algebras over commutative rings, as well as some basic facts from the classical representation theory of associative algebras over fields. The classical reference for Coxeter groups is N. Bourbaki [Bo]. Especially suited for the things that we need is [CR], $\S 64,68$.

2. Finite Coxeter groups of classical type. A Coxeter group $W$ is a group given by a presentation of a very special form: We have a finite set of generators $S \subseteq W$ which we write in the form $S=\left\{s_{i} \mid i \in I\right\}$ for some finite index set $I$, and the defining relations are just relations of the form $\left(s_{i} s_{j}\right)^{m(i, j)}=1$ where $i, j \in I$ and $m(i, j)>1$ for $i \neq j, m(i, i)=1$. Since each generator is equal to its inverse, we can rewrite the set of relations $\left(s_{i} s_{j}\right)^{m(i, j)}=1$, for $i \neq j$, equivalently in the form of the equalities $s_{i} s_{j} \cdots=s_{j} s_{i} \cdots$, with $m(i, j)$ factors on both sides. These relations will be called braid relations, or homogeneous relations. Thus, a Coxeter group is a group generated by a set of involutions which satisfy a defining set of homogeneous relations (where the number of factors on each side of the equation has been prescribed).

We shall assume throughout that the integers $m(i, j)$ are chosen so that $W$ is a finite group. This is the case if and only if the matrix $(-\cos (\pi / m(i, j)))_{i, j \in I}$ is positive-definite. We only remark that all matrices $(m(i, j))$ with this property can be classified, and that this classification leads to the well-known list of classical types $A_{n-1}, B_{n}, D_{n}$ (for $n \geq 1$ ); of exceptional types $G_{2}, F_{4}, E_{6}, E_{7}, E_{8}$; and of non-crystallographic types $I_{2}(p)$ (for $p \geq 5$, $p \neq 6), H_{3}$ and $H_{4}$. Moreover, every such group has a geometrical description as a finite subgroup of the orthogonal group of an Euclidean space of dimension $|I|$, where the generators $s_{i}$ are represented by reflections. We shall not need this classification result in this paper, and we shall only be dealing with the classical types $A_{n-1}, B_{n}, D_{n}$ whose diagrams are given as follows.

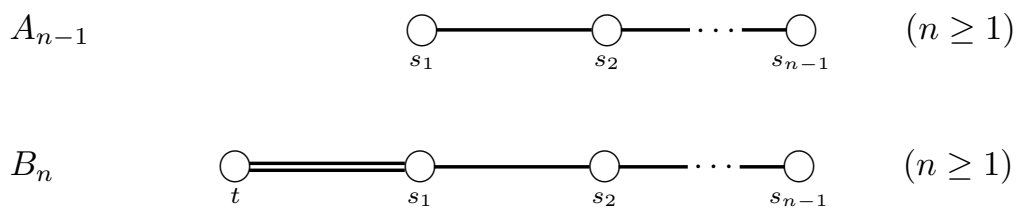




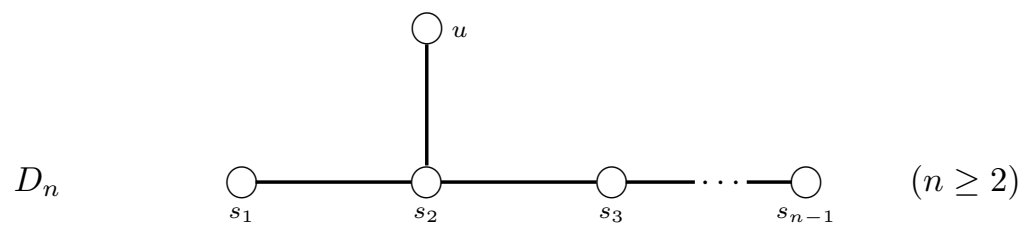

These diagrams encode the relations among the generators by the following scheme. The nodes of the diagram are in bijection with the generators of $W$. If two generators label nodes which are not directly linked to each other then their product has order 2 (that is, they commute with each other), if they are joined by a single bond then the product has order 3 , in the case of a double bond (which here only occurs for type $B_{n}$ ) the order is 4 .

On $W$, we have a length function defined as follows. Let $w \in W$. Then it is possible to write $w$ as a product of generators, $w=s_{i_{1}} \cdots s_{i_{k}}$ say, where $k$ is a non-negative integer and $i_{1}, \ldots, i_{k} \in I$. (Note that we don't have to take into account the inverses of the generators.) If we choose $k$ minimal with this property, then the corresponding product of generators will be called a reduced expression for $w$ and $k$ will be the length of $w$, denoted $l(w)$. Clearly, we have $l(1)=0$ and $l\left(s_{i}\right)=1$ for all $i \in I$. On the other hand, for any $w \in W$ and $i \in I$ we have either $l\left(s_{i} w\right)=l(w)+1$ or $l\left(s_{i} w\right)=l(w)-1$ (and similarly for right multiplication by a generator). A key property of a Coxeter group is the fact (Matsumoto's Theorem, see [CR], (64.20)) that any two reduced expressions for a given element can be transformed into each other by applying a finite sequence of braid relations.

We will see below how we can find reduced expressions for the elements of the Coxeter groups of classical type $A_{n-1}, B_{n}$ and $D_{n}$.

2.1. Normal form for type $A_{n-1}$. We consider the Coxeter group of type $A_{n-1}(n \geq 1)$ with generators and relations given by the above diagram. Let us take an arbitrary product of generators $s_{i_{1}} \cdots s_{i_{r}}$, for some $r \geq 0$. We now use the braid relations and the relations $s_{i}^{2}=1$ as rewriting rules for subexpressions, that is, if our product contains a subword which looks like the left hand side of any one of these relations then we can replace it by the right hand side, and repeat this process. By a simple induction on $r$ it then follows that we can rewrite our expression in the form $s_{i_{1}} \cdots s_{i_{r}}=s_{j_{1}} \cdots s_{j_{t}}$ where $j_{1}, \ldots, j_{t} \in I$ for some $0 \leq t \leq r$, and at most one factor in the product on the right hand side equals $s_{n-1}$. Now let us define the set

$$
\mathcal{R}_{n}^{+}:=\left\{1, s_{n-1}, s_{n-1} s_{n-2}, \ldots, s_{n-1} s_{n-2} \cdots s_{1}\right\} .
$$

(This definition works for all $n \geq 1$, with $\mathcal{R}_{1}^{+}=\{1\}$.) Note that $\mathcal{R}_{n}^{+}$has cardinality $n$. A second induction then implies that an arbitrary product of $s_{i}$ 's can be written in the form $r_{1} \cdots r_{n}$ with $r_{i} \in \mathcal{R}_{i}^{+}$for all $i$. Since there are only $n$ ! expressions of this form we conclude that our group has order at most $n$ !.

On the other hand, we know that the symmetric group $\mathcal{S}_{n}$ on $n$ letters is generated by the transpositions $\sigma_{i}(1 \leq i \leq n-1)$ which interchange the letters $i, i+1$ and leave all others unchanged, and that these elements $\sigma_{i}$ satisfy the defining relations for type $A_{n-1}$. Thus we conclude that $\mathcal{S}_{n}$ is in fact a realization of the Coxeter group of type $A_{n-1}$. It 
also follows that the above expressions of the elements of $\mathcal{S}_{n}$ are reduced expressions; we shall call them the normal form for the elements of $\mathcal{S}_{n}$.

2.2. Normal form for type $B_{n}$. We consider the Coxeter group of type $B_{n}(n \geq 1)$ and denote it by $W_{n}$. In order to obtain a normal form for its elements we define

$$
t_{j}:=s_{j} s_{j-1} \cdots s_{1} t s_{1} \cdots s_{j-1} s_{j} \in W_{n} \quad \text { for } \quad j=0,1,2, \ldots
$$

By a similar induction as before we see that every element of $W_{n}$ can be written as a product of factors $s_{i}(1 \leq i \leq n-1)$ and $t_{j}(0 \leq j \leq n-1)$ in such a way that $s_{n-1}$ and $t_{n-1}$ occur at most once, and if one of these two elements occurs the other doesn't. Now let us define the set

$$
\mathcal{R}_{n}:=\left\{s_{n-1} \cdots s_{i+1}, s_{n-1} s_{n-2} \cdots s_{i+1} t_{i} \mid 0 \leq i \leq n-1\right\} .
$$

This set has cardinality $2 n$ (where $\mathcal{R}_{1}=\{1, t\}$ ). As above, we can then conclude that every element in $W_{n}$ can be written in the form $r_{1} \cdots r_{n}$ with $r_{i} \in \mathcal{R}_{i}$ for all $i$. Since there are only $2 \cdot 4 \cdots 2 n=2^{n} n$ ! expressions of this form, we deduce that our group has order at most $2^{n} n$ !.

On the other hand, we know that the hyperoctahedral group, that is, the symmetry group of an $n$-dimensional cube, is a quotient of $B_{n}$. A good way to think about this group is as follows. We consider the symmetric group of degree $2 n$ where we denote the objects being permuted by $\left\{n, \ldots, 1,1^{\prime}, \ldots, n^{\prime}\right\}$. Then the hyperoctahedral group is the subgroup of all elements which commute with the map $i \mapsto i^{\prime}, i^{\prime} \mapsto i(1 \leq i \leq n)$. Thus, it is generated by the permutations $t=\left(1,1^{\prime}\right), s_{1}=(1,2)\left(1^{\prime}, 2^{\prime}\right), \ldots, s_{n-1}=$ $(n-1, n)\left((n-1)^{\prime}, n^{\prime}\right)$. It is readily checked that these elements indeed satisfy the defining relations for a Coxeter group of type $B_{n}$, and that it has the correct order.

It can be further shown that $t_{i}$ commutes with $t_{j}$ for all $i, j$, hence the set of elements $\left\{t_{i_{1}} \cdots t_{i_{r}} \mid 0 \leq i_{1}<\ldots<i_{r} \leq n-1\right\}$ is a subgroup of $W_{n}$ isomorphic to the direct product of $n$ copies of the cyclic group $C_{2}$ of order 2. This subgroup clearly is invariant under conjugation by all generators. On the other hand, the subgroup of $W_{n}$ generated by $\left\{s_{1}, \ldots, s_{n-1}\right\}$ is isomorphic to the symmetric group $\mathcal{S}_{n}$, and we obtain a decomposition of $W_{n}$ as the wreath product $C_{2} \prec \mathcal{S}_{n}$ where $\mathcal{S}_{n}$ acts on $C_{2}^{n}$ by permutation of the components.

2.3. Normal form for type $D_{n}$. The Coxeter group of type $D_{n}$ (for $n \geq 2$ ) is best described as a subgroup of index 2 in the Coxeter group $W_{n}$ of type $B_{n}$ considered before. First note that the defining relations for $W_{n}$ show that there exists a unique group homomorphism $\varepsilon: W_{n} \rightarrow\{1,-1\}$ such that $\varepsilon(t)=-1$ and $\varepsilon\left(s_{i}\right)=1$ for all $i$. The kernel of this map, which we will denote by $W_{n}^{\prime}$, is generated by the elements $u:=t s_{1} t, s_{1}, \ldots, s_{n-1}$, which satisfy the relations given by the above Dynkin diagram of type $D_{n}$. Thus, the group $W_{n}^{\prime}$ is seen to be a quotient of the Coxeter group of type $D_{n}$. Formally, this also works for $n=1$ where it leads to the convention that $W_{n}^{\prime}=\{1\}$ and $u$ is considered as being equal to the identity element. On the other hand, one can check (using the Reidemeister-Schreier procedure for finding a presentation of a subgroup of a finitely presented group) that the subgroup of $W_{n}$ generated by $\left\{u, s_{1}, \ldots, s_{n-1}\right\}$ indeed is a realization of the Coxeter group of type $D_{n}$. An element of $W_{n}$ belongs to $W_{n}^{\prime}$ if and 
only if it can be written as a product of various generators $t, s_{1}, \ldots, s_{n-1}$ with an even number of $t$ 's.

Let us also derive a normal form for the elements in $W_{n}^{\prime}$, directly in terms of the generators $\left\{u, s_{1}, \ldots, s_{n-1}\right\}$. We have $W_{n}=W_{n}^{\prime} \cup W_{n}^{\prime} t$ and $W_{n}=\bigcup_{r \in \mathcal{R}_{n}} W_{n-1} r$. These two equalities imply that an element of $W_{n}^{\prime}$ either is of the form $w r$ (with $w \in W_{n-1}^{\prime}$ and where $r \in \mathcal{R}_{n}$ does not contain $t$ ) or of the form $w t r$ (with $w \in W_{n-1}^{\prime}$ and where $r \in \mathcal{R}_{n}$ does contain $t$ ). We can rewrite such expressions in terms of the generators of $W_{n}^{\prime}$ and are lead to define the set

$$
\mathcal{R}_{n}^{\prime}:=\left\{s_{n-1} s_{n-2} \cdots s_{i+1}, s_{n-1} s_{n-2} \cdots s_{2} u s_{1} \cdots s_{i} \mid 0 \leq i \leq n-1\right\} .
$$

This set has cardinality $2 n$ (where $\mathcal{R}_{2}^{\prime}=\left\{1, u, s_{1}, u s_{1}\right\}$ and, as a convention, $\mathcal{R}_{1}^{\prime}=\{1\}$ ). Then every element in $W_{n}^{\prime}$ can be written uniquely in the form $r_{1} \cdots r_{n}$ with $r_{i} \in \mathcal{R}_{i}^{\prime}$ for all $i$, and these expressions are reduced. (There are exactly $1 \cdot 4 \cdot 6 \cdots 2 n=2^{n-1} n$ ! such expressions.)

To summarize the above descriptions we introduce the following notation. The symbol $X_{n}$ (for $n \geq 1$ ) denotes one of the above classical types $A_{n-1}, B_{n}$, or $D_{n}$. Then $W\left(X_{n}\right)$ is the corresponding Coxeter group and $\mathcal{R}\left(X_{n}\right)$ the subset of $W\left(X_{n}\right)$ called $\mathcal{R}_{n}^{+}$for type $A_{n-1}, \mathcal{R}_{n}$ for type $B_{n}$, and $\mathcal{R}_{n}^{\prime}$ for type $D_{n}$. For $n \geq 2$, the group $W\left(X_{n-1}\right)$ is naturally embedded into $W\left(X_{n}\right)$, and the set $\mathcal{R}\left(X_{n}\right)$ is nothing but the set of so-called distinguished right coset representatives of $W\left(X_{n}\right)$ with respect to $W\left(X_{n-1}\right)$. Thus, it satisfies the following properties (cf. [CR], (64.38)).

$* \mathcal{R}\left(X_{1}\right)=W\left(X_{1}\right)$.

* If $n \geq 2$ then every element $w \in W\left(X_{n}\right)$ can be written uniquely in the form $w=w^{\prime} r$ with $w^{\prime} \in W\left(X_{n-1}\right)$ and $r \in \mathcal{R}\left(X_{n}\right)$, and we have $l(w)=l\left(w^{\prime}\right)+l(r)$

In particular, every element $w \in W\left(X_{n}\right)$ can be written uniquely in the form $w=r_{1} \cdots r_{n}$ with $r_{i} \in \mathcal{R}\left(X_{i}\right)$ for all $i$ (and these expressions are reduced). Thus we have a uniform inductive description of the elements in Coxeter groups of classical type.

3. Conjugation in the Coxeter groups of classical type. We shall now use the above inductive description as an essential tool to derive the crucial result about the form of the conjugacy classes in Coxeter groups of classical types. Let again $n \geq 1$ and $X_{n}$ one of the types $A_{n-1}, B_{n}$ or $D_{n}$. Recalling the definition of the set $\mathcal{R}\left(X_{n}\right)$ we see that each of its elements (for $n \geq 2$ ) has a very special form: It is either a product of $s_{n-1}$ and an element lying in $W\left(X_{n-1}\right)$ or it is equal to 1 , respectively $t_{n-1}=s_{n-1} \cdots s_{1} t s_{1} \cdots s_{n-1}$ (in type $B_{n}$ ) or $u_{n-1}:=s_{n-1} \cdots s_{2} u s_{1} s_{2} \cdots s_{n-1}$ (in type $D_{n}$ ). In order to obtain a uniform description we introduce subsets $\mathcal{D}\left(X_{n}\right) \subseteq \mathcal{R}\left(X_{n}\right)$ for all $n \geq 1$ as follows.

Definition. If $n=1$ we let $\mathcal{D}\left(X_{1}\right):=\mathcal{R}\left(X_{1}\right)=W\left(X_{1}\right)$. For $n \geq 2$ we give the definition case by case.

Type $A_{n-1}: \quad \mathcal{D}\left(X_{n}\right):=\left\{1, s_{n-1}\right\}$.

Type $B_{n}: \mathcal{D}\left(X_{n}\right):=\left\{1, s_{n-1}, t_{n-1}\right\}$.

Type $D_{n} \quad: \quad \mathcal{D}\left(X_{n}\right):=\left\{1, s_{n-1}, u_{n-1}\right\}$ for $n \geq 3$ and $\mathcal{D}\left(X_{2}\right):=W\left(X_{2}\right)$.

(Recall that $t_{n-1}=s_{n-1} \cdots s_{1} t s_{1} \cdots s_{n-1}$ respectively $u_{n-1}=s_{n-1} \cdots s_{2} u s_{1} s_{2} \cdots s_{n-1}$.) 
The form of the elements in the sets $\mathcal{D}\left(X_{n}\right)$ and $\mathcal{R}\left(X_{n}\right)$ then immediately implies the following result.

LEMma 3.1 (Double coset decomposition). Let $n \geq 2$ and $\mathcal{D}\left(X_{n}\right)$ as defined above. Then every element $w \in W\left(X_{n}\right)$ can be written in the form $w=w_{1} d w_{2}$ with $w_{1}, w_{2} \in$ $W\left(X_{n-1}\right), d \in \mathcal{D}\left(X_{n}\right)$ and $l(w)=l\left(w_{1}\right)+l(d)+l\left(w_{2}\right)$.

Now recall the following general definition from $[\mathrm{GP}]$. Let $W$ be an arbitrary Coxeter group, with generating set $S=\left\{s_{i} \mid i \in I\right\}$. Given $w, w^{\prime} \in W$ we write $w \longrightarrow w^{\prime}$ if there exists a sequence of elements $w=w_{1}, \ldots, w_{m}=w^{\prime}$ (for some $m \geq 1$ ) and indices $i_{1}, \ldots, i_{m-1}$ such that $w_{k+1}=s_{i_{k}} w_{k} s_{i_{k}}$ and $l\left(w_{k+1}\right) \leq l\left(w_{k}\right)$ for all $k$. Thus, the relation $w \longrightarrow w^{\prime}$ means that we can conjugate $w$ to $w^{\prime}$ step by step by a sequence of generators such that the length of the elements remains the same at each step or decreases.

Now we can state our crucial result.

Proposition 3.2 (Cf. [GP], Proposition 2.3). Let $n \geq 1$ and $w \in W\left(X_{n}\right)$. Then there exist some $d_{i} \in \mathcal{D}\left(X_{i}\right)$, for $1 \leq i \leq n$, such that $w \rightarrow d_{1} \cdots d_{n}$ and such that the generators performing the sequence of conjugations lie in $W\left(X_{n-1}\right)$.

Proof. For $n=1$ there is nothing to prove due to the definition of $\mathcal{D}\left(X_{1}\right)$. Now let $n \geq 2$ and $w \in W\left(X_{n}\right)$. By Lemma 3.1 we can write $w=w^{\prime} d_{n} w^{\prime \prime}$ with $w^{\prime}, w^{\prime \prime} \in W\left(X_{n-1}\right)$ and $d_{n} \in \mathcal{D}\left(X_{n}\right)$. Taking any reduced expression for $w^{\prime \prime}$ and conjugating step by step by the generators involved in this reduced expression yields that

$$
w \longrightarrow w_{n-1} d_{n} \text { for some } w_{n-1} \in W\left(X_{n-1}\right) .
$$

If $n=2$ we are done, otherwise we can again write $w_{n-1}=w^{\prime} d_{n-1} w^{\prime \prime}$ with $w^{\prime}, w^{\prime \prime} \in$ $W\left(X_{n-2}\right)$ and $d_{n-1} \in \mathcal{D}\left(X_{n-1}\right)$.

Now it readily follows using the braid relations that, for $n \geq 3$, each element in $\mathcal{D}\left(X_{n}\right)$ commutes with the generators for $W\left(X_{n-2}\right)$, with the only exception that in type $D_{n}$ we only have $u_{n-1} u=s_{1} u_{n-1}$ and $u_{n-1} s_{1}=u u_{n-1}$. In any case the following relation holds. For each generator $x$ of $W\left(X_{n-2}\right)$ and each $d \in \mathcal{D}\left(X_{n}\right)$ there exists some generator $x^{\prime}$ of $W\left(X_{n-2}\right)$ such that $x d=d x^{\prime}$.

Returning to our above equation $w_{n-1}=w^{\prime} d_{n-1} w^{\prime \prime}$ we take a reduced expression for $w^{\prime \prime}$, conjugate step by step by the generators involved in it, and conclude that

$$
w_{n-1} d_{n}=w^{\prime} d_{n-1} w^{\prime \prime} d_{n} \longrightarrow w_{n-2} d_{n-1} d_{n} \text { for some } w_{n-2} \in W\left(X_{n-2}\right) .
$$

Continuing in this way, we obtain the desired result after finitely many steps. Note that the conjugating elements all lie in $W\left(X_{n-1}\right)$. This completes the proof.

An element of the form $d_{1} \cdots d_{n}$, with $d_{i} \in \mathcal{D}\left(X_{i}\right)$ for all $i$, will be called an element in signed block form. Such elements play an essential role later on in our classification of Markov traces. If we group together consecutive factors $s_{i}$ we obtain a decomposition of our given element as a product of "blocks". To explain this and the notion of "positive blocks" and "negative blocks" in more detail we consider in turn the classification of conjugacy classes for type $A_{n-1}, B_{n}$ and $D_{n}$.

3.3. Conjugacy classes for type $A_{n-1}$. By Proposition 3.2, any element of $W\left(A_{n-1}\right)$ (for $n \geq 1$ ) is conjugate to an element of the form $d_{1} \cdots d_{n}$ with $d_{i} \in \mathcal{D}\left(A_{i-1}\right)$ for all $i$. 
Let us fix such an element. Since each factor $d_{i}$ either equals 1 or equals $s_{i-1}$ we see that our element is uniquely determined by the sequence of non-negative integers defined by the condition that $d_{i+1}=1$ if and only if $i$ belongs to that sequence. Denote this sequence by $0=m_{1}<\ldots<m_{r}$, and let us also define $\lambda:=\left(\lambda_{1}, \ldots, \lambda_{r}\right)$ where $\lambda_{i}:=m_{i+1}-m_{i}$ for $i=1, \ldots, r$ (with the convention that $m_{r+1}=n$ ). Note that the entries of $\lambda$ are all positive and their sum equals $n$ (that is, $\lambda$ forms a composition of $n$ ). The integers $m_{i}$ are determined from $\lambda$ by the rule $m_{i}=\lambda_{1}+\ldots+\lambda_{i-1}$ for all $i \geq 1$. With this notation, we now have $d_{1} \cdots d_{n}=w(\lambda)$ where

$$
w(\lambda):=b^{+}\left(m_{1}, \lambda_{1}\right) \cdots b^{+}\left(m_{r}, \lambda_{r}\right)
$$

and where we define, for any $m \geq 0$ and $d \geq 1$,

$$
b^{+}(m, d):=s_{m+1} \cdots s_{m+d-1} .
$$

Under the isomorphism $W\left(A_{n-1}\right) \cong \mathcal{S}_{n}$, such an element $b^{+}(m, d)$ corresponds to the $d$-cycle $(m+1, \ldots, m+d)$ which permutes the $d$ numbers in the interval between $m+1$ and $m+d$ cyclically and leaving all other numbers unchanged. We call it a positive block of length $d$ and starting at $m$; the interval $\{m+1, \ldots, m+d\}$ will be called the underlying index set. The braid relations imply that two positive blocks commute with each other if the underlying index sets are disjoint.

Let $\lambda$ and $\lambda^{\prime}$ be two compositions of $n$ which are obtained from each other by permuting the parts. Assume at first that only two consecutive parts have been permuted. Then there exists an element $y \in W\left(A_{n-1}\right)$ such that $y w(\lambda) y^{-1}=w\left(\lambda^{\prime}\right)$ and $l(y w(\lambda))=l(y)+l(w(\lambda))$. The element $y$ can be written down explicitly as a reduced expression in the generators (see [GP], Proposition 2.4(a)). The relevance of the length condition will become clear later on. Applying this relation repeatedly eventually proves that every element of $W\left(A_{n-1}\right)$ is conjugate to an element $w(\lambda)$ where $\lambda$ is a partition of $n$, that is, a composition with parts ordered by increasing length. Since conjugation in $\mathcal{S}_{n}$ never changes the cycle type of elements, we have thus recovered the well-known classification of conjugacy classes by partitions of $n$, and we have described canonically reduced expressions for a set of representatives in the various conjugacy classes. We remark that theses representatives have minimal length in their classes (see [GP], (2.6)).

For example, the classes for $W\left(A_{3}\right) \cong \mathcal{S}_{4}$ are parametrized by the partitions (1111), (112), (13), (22), and (4). The corresponding representatives of minimal length are given by $1, s_{3}, s_{2} s_{3}, s_{1} s_{3}$ and $s_{1} s_{2} s_{3}$, respectively.

3.4. Conjugacy classes for type $B_{n}$. Let us denote $W_{n}=W\left(B_{n}\right)$ for $n \geq 1$. Using Proposition 3.2, we can proceed similarly as for type $A_{n-1}$, with the only difference that we also have to take into account the double coset representatives $t_{i}$. Therefore we define, for any $m \geq 0$ and $d \geq 1$,

$$
b^{-}(m, d):=t_{m} s_{m+1} \cdots s_{m+d-1}
$$

and call this element a negative block of length $d$ and starting at $m$, with underlying index set $\{m+1, \ldots, m+d\}$. (Positive blocks are defined in exactly the same way as for type $A_{n-1}$.) We can now conclude that every element in $W_{n}$ is conjugate to an element of the 
form

$$
w(\lambda, \varepsilon):=b^{\varepsilon_{1}}\left(m_{1}, \lambda_{1}\right) \cdots b^{\varepsilon_{r}}\left(m_{r}, \lambda_{r}\right),
$$

where $\lambda=\left(\lambda_{1}, \ldots, \lambda_{r}\right)$ is a composition of $n, \varepsilon=\left(\varepsilon_{1}, \ldots, \varepsilon_{r}\right)$ is a vector of signs, and $m_{i}$ is the sum of the first $i-1$ parts of $\lambda$ (as before). Using the description of $W_{n}$ as a certain subgroup of $S_{2 n}$ described in $(2.2)$, a positive block $b^{+}(m, d)$ is given by the " $d$-cycle" $(m+1, \ldots, m+d)\left((m+1)^{\prime}, \ldots,(m+d)^{\prime}\right)$ while the corresponding negative block equals $\left(m+1,(m+1)^{\prime}\right) b^{+}(m, d)$. (Thus, multiplication by $t_{m}=\left(m+1,(m+1)^{\prime}\right)$ is considered as a "sign change" for a permutation.) Again the braid relations imply that any two blocks commute with each other if the underlying index sets are disjoint.

We conjugate our element further so as to obtain a signed block structure where the positive and negative blocks are arranged in some prescribed order. This is done as follows. In a first step, we have $w(\lambda, \varepsilon) \longrightarrow w\left(\lambda^{\prime}, \varepsilon^{\prime}\right)$ where $\lambda^{\prime}, \varepsilon^{\prime}$ are obtained by rearranging $\lambda, \varepsilon$ such that all negative parts are in the beginning and ordered by increasing length (see [GP], Proposition $2.4(\mathrm{~b}, \mathrm{c}))$. Thus, $\varepsilon^{\prime}=(-1, \ldots,-1,1, \ldots, 1)$ with $m$ negative signs say. If the sign vector has such a form, we also denote the element $w\left(\lambda^{\prime}, \varepsilon^{\prime}\right)$ by $w(\alpha, \beta)$ where $\alpha$ is the sequence consisting of the first $m$ parts of $\lambda^{\prime}$ (with corresponding negative sign) and $\beta$ of the remaining parts. The sequence $\alpha$ already forms a partition, while the parts of $\beta$ may have some arbitrary order. Thus, we see that for each $w \in W_{n}$ we have $w \longrightarrow w(\alpha, \beta)$ where $\alpha$ is a partition and $\beta$ is a composition such that the total sum of their parts equals $n$. Finally, the positive blocks (corresponding to the parts of $\beta$ ) can be arranged in increasing order in exactly the same way as this was done in type $A_{n-1}$.

Thus, given partitions $\alpha=\left(\alpha_{1}, \ldots, \alpha_{r}\right)$ and $\beta=\left(\beta_{1}, \ldots, \beta_{s}\right)$ such that $\sum_{i} \alpha_{i}+$ $\sum_{j} \beta_{j}=n$, we let $m_{i}:=\alpha_{1}+\ldots+\alpha_{i-1}$ and $n_{j}:=m_{r+1}+\beta_{1}+\ldots+\beta_{j-1}$ for all $i, j \geq 1$; then the corresponding representative is given by

$$
w(\alpha, \beta)=b^{-}\left(m_{1}, \alpha_{1}\right) \cdots b^{-}\left(m_{r}, \alpha_{r}\right) b^{+}\left(n_{1}, \beta_{1}\right) \cdots b^{+}\left(n_{s}, \beta_{s}\right) .
$$

In this way we can recover the classification of conjugacy classes by pairs of partitions of $n$ (see [Ca]). We have described reduced expressions for a set of canonical representatives in the various conjugacy classes. Again, these representatives have minimal length in their classes (see [GP], (2.7)).

For example, the classes for $W\left(B_{3}\right)$ are parametrized by the double partitions $(-, 111)$, $(1,11),(11,1),(111,-),(-, 12),(2,1),(1,2),(12,-),(-, 3)$, and $(3,-)$. The corresponding representatives of minimal length are given by $1, t, t t_{1}, t t_{1} t_{2}, s_{2}, t s_{1}, t s_{2}, t t_{1} s_{2}, s_{1} s_{2}$, and $t s_{1} s_{2}$, respectively.

3.5. Conjugacy classes for type $D_{n}$. We use the notation of (2.3) and consider the Coxeter group $W_{n}^{\prime}$ of type $D_{n}$ as a subgroup of index 2 in the Coxeter group $W_{n}$ of type $B_{n}$ (for $n \geq 2$ ). The description of the classes of $W_{n}^{\prime}$ is then in terms of the results obtained above in (3.4). Since $W_{n}^{\prime}$ is a normal subgroup, it is a union of conjugacy classes of $W_{n}$. Let $(\alpha, \beta)$ be a pair of partitions such that the total sum of their parts equals $n$, and let $C(\alpha, \beta)$ be the corresponding class in $W_{n}$. Then $C(\alpha, \beta)$ belongs to $W_{n}^{\prime}$ if and only if the number of parts of $\alpha$ (corresponding to the negative blocks) is even. Assume that this is the case. Then $C(\alpha, \beta)$ either is one conjugacy class of $W_{n}^{\prime}$ or it splits up into two classes. The latter happens if and only if $\alpha$ is empty and all parts of $\beta$ are even; in 
this case, the two classes are mapped to each other under conjugation by the generator $t$. (For these results, see [Ca].)

Taking the elements $w(\alpha, \beta)$ obtained in (3.4) and rewriting them in terms of the generators of $W_{n}^{\prime}$ will yield canonical representatives of minimal length in the classes of $W_{n}^{\prime}$. This is done as follows. We define a negative block of length $d \geq 1$ and starting at $m \geq 0$ by

$$
b^{-}(m, d)^{\prime}:=u_{m} s_{m+1} \cdots s_{m+d-1} \in W_{n}^{\prime},
$$

with the convention that $u_{0}=1$. (Positive blocks are defined in exactly the same way as before.) Given compositions $\alpha$ and $\beta$ such that the total sum of their parts equals $n$ we define the element $w(\alpha, \beta)^{\prime}$ in a similar way as in (3.4) as the product of consecutive negative and positive blocks corresponding to the sequence of parts in $\alpha$ and $\beta$. The relation $u=t s_{1} t$ and the definition of $u_{i}$ show that $u_{i}=t t_{i}$ for all $i \geq 0$. Inserting this into the above expressions for negative blocks we conclude that $w(\alpha, \beta)^{\prime}$ is either equal to $w(\alpha, \beta)$ (defined above for type $\left.B_{n}\right)$ or to $t w(\alpha, \beta) t$.

Now, if $\alpha$ is empty and all parts of $\beta$ are even then $w(\alpha, \beta)^{\prime}$ is a product of positive blocks, starting with $s_{1} \cdots s_{d-1}$, for some $d \geq 2$. Conjugation by $t$ then leads to an expression of the same form where $s_{1}$ has been replaced by $u$. These two elements are representatives for the two classes into which $C(\alpha, \beta)$ splits up in $W_{n}^{\prime}$. For example, if $n$ is even, then the two elements $s_{1} s_{2} \cdots s_{n-1}$ and $u s_{2} \cdots s_{n-1}$ are transformed into each other by conjugation with $t$ but they are not conjugate in $W_{n}^{\prime}$. Note that if $\alpha$ is not empty, then $w(\alpha, \beta)$ and $t w(\alpha, \beta) t$ are in the same conjugacy class in $W_{n}^{\prime}$.

Using these relations and a careful comparison of conjugation in $W_{n}$ and in $W_{n}^{\prime}$ we arrive at the following conclusion (see [GP], (2.8), and [Pf], Section 4.1, for details). For each $w \in W_{n}^{\prime}$ we either have $w \longrightarrow w(\alpha, \beta)^{\prime}$ where $\alpha$ is a partition with an even number of parts and $\beta$ a composition such that the total sum of their parts equals $n$ or, possibly, $w \longrightarrow t w(\alpha, \beta)^{\prime} t$ if $\alpha$ is empty and all parts of $\beta$ are even. In both cases, the positive blocks can be arranged in increasing order by the same reasoning as in type $A_{n-1}$. Thus, we obtain canonical representatives of minimal length in the conjugacy classes of $W_{n}^{\prime}$.

For example, in $W\left(D_{4}\right)$, the above classification yields that there are 9 classes which are invariant under conjugation by $t$; these are parametrized by the double partitions $(-, 1111),(11,11),(1111,-),(-, 112),(12,1),(11,2),(22,-),(-, 13)$, and $(13,-)$. The corresponding representatives of minimal length are given by $1, u_{1}, u_{1} u_{2} u_{3}, s_{3}, u_{1} s_{2}$, $u_{1} s_{3}, s_{1} u_{2} s_{3}, s_{2} s_{3}$, and $u_{1} s_{2} s_{3}$, respectively. Moreover, we have the two double partitions $(-, 22)$ and $(-, 4)$ for which we have two classes each, with representatives of minimal length given by $s_{1} s_{3}, u s_{3}$ and $s_{1} s_{2} s_{3}, u s_{2} s_{3}$, respectively.

In summary, the above arguments were used in $[\mathrm{GP}]$ to prove the following basic results for Coxeter groups of classical type.

Theorem 3.6 (See [GP], Theorem 1.1). Let $W$ be a finite Coxeter group, $C$ a conjugacy class in $W$, and $C_{\text {min }}$ be the set of elements of minimal length in $C$.

(a) For each $w \in C$ there exists some $w^{\prime} \in C_{\text {min }}$ such that $w \longrightarrow w^{\prime}$, that is, there exists a sequence of elements $w=w_{1}, \ldots, w_{m}=w^{\prime}($ for some $m \geq 1)$ and indices $i_{1}, \ldots, i_{m-1}$ such that $w_{k+1}=s_{i_{k}} w_{k} s_{i_{k}}$ and $l\left(w_{k+1}\right) \leq l\left(w_{k}\right)$ for all $k$. 
(b) If $w, w^{\prime} \in C_{\min }$ then $w \sim w^{\prime}$, that is, there exists a sequence $w=w_{0}, \ldots, w_{m}=w^{\prime}$ (for some $m \geq 1$ ) and elements $x_{1}, \ldots, x_{m} \in W\left(X_{n}\right)$ such that $l\left(w_{k}\right)=l\left(w_{k+1}\right), w_{k+1}=$ $x_{k} w_{k} x_{k}^{-1}$, and $l\left(x_{k} w_{k}\right)=l\left(x_{k}\right)+l\left(w_{k}\right)$ or $l\left(w_{k} x_{k}^{-1}\right)=l\left(w_{k}\right)+l\left(x_{k}^{-1}\right)$ for all $k$.

4. Iwahori-Hecke algebras. Let $W$ be a finite Coxeter group with generating set $S=\left\{s_{i} \mid i \in I\right\}$ as introduced in Section 2. Let $A$ be a commutative ring with identity and fix a collection of invertible elements $q_{i} \in A(i \in I)$ such that $q_{i}=q_{j}$ whenever $s_{i}$ and $s_{j}$ are conjugate in $W$. (The latter condition holds for $s_{i}$ and $s_{j}$ which are joined on the Dynkin diagram if and only if the integer $m(i, j)$ is odd.) The associated IwahoriHecke algebra $H$ is a free $A$-module with a basis labelled by the elements of $W$, say $\left\{g_{w} \mid w \in W\right\}$, and where the multiplication of two basis elements is determined by "deforming" the multiplication in the Coxeter group using the parameters $q_{i}(i \in I)$. To simplify notation, let us write $g_{i}=g_{s_{i}}$ for $i \in I$. A convenient way to describe the multiplication is then given by the following two rules.

If $w \in W$ and $w=s_{i_{1}} \cdots s_{i_{k}}$ is any reduced expression then $g_{w}=g_{i_{1}} \cdots g_{i_{k}}$.

If $i \in I$ then $g_{i}^{2}=q_{i} \cdot 1_{H}+\left(q_{i}-1\right) g_{i}$.

It is a non-trivial fact that this indeed is a well-defined multiplication and gives $H$ the structure of an associative algebra over $A$. The identity element $1_{H}$ is the basis element corresponding to $w=1$. (A proof is sketched in [Bo], Ch. IV, §2, Exercise 23; for full details see [CR], Proposition 68.1. Note that this works even if $W$ is not finite.) If the $q_{i}$ are all equal to 1 then the second rule simply becomes $g_{i}^{2}=1$ for all $i \in I$; in this case, $H$ is nothing but the group algebra of $W$ over $A$.

The above relations show that $H$ is generated by the elements $g_{i}(i \in I)$. Moreover, for $i \neq j$, we have $g_{i} g_{j} g_{i} \cdots=g_{j} g_{i} g_{j} \cdots$ (with $m(i, j)$ factors on both sides) since $s_{i} s_{j} s_{i} \cdots$ and $s_{j} s_{i} s_{j} \cdots$ (with $m(i, j)$ factors) are reduced expressions and equal in $W$. It is then not difficult to see (cf. [CR], Proposition 68.8) that these homogeneous relations together with the above quadratic relations for the generators $g_{i}$ form in fact a set of defining relations for $H$ (as an associative $A$-algebra with identity).

It may be worthwhile to be a bit more explicit about the multiplication in $H$. Let us multiply two arbitrary basis elements $g_{v}$ and $g_{w}$ for $v, w \in W$. For this purpose, we take a reduced expression for one of them, say $v=s_{i_{1}} \cdots s_{i_{k}}$. By the first rule above we have $g_{v}=g_{i_{1}} \cdots g_{i_{k}}$. Thus, we are reduced to the case where $v=s_{i}$ for some $i \in I$. If $l\left(s_{i} w\right)=l(w)+1$ then $s_{i} w$ also is reduced and we have, again by the first rule above, that $g_{i} g_{w}=g_{s_{i} w}$. If $l\left(s_{i} w\right)=l(w)-1$ we take a reduced expression for $s_{i} w$, say $s_{i} w=s_{j_{1}} \cdots s_{j_{t}}$. Then $w=s_{i}\left(s_{i} w\right)=s_{i} s_{j_{1}} \cdots s_{j_{t}}$ also is reduced and we can compute that

$$
g_{w}=g_{s_{i}\left(s_{i} w\right)}=g_{s_{i} s_{j_{1}} \cdots s_{j_{t}}}=g_{i}\left(g_{j_{1}} \cdots g_{j_{t}}\right)=g_{i} g_{s_{i} w} .
$$

We conclude that

$$
g_{i} g_{w}=g_{i}^{2} g_{s_{i} w}=q_{i} g_{s_{i} w}+\left(q_{i}-1\right) g_{i} g_{s_{i} w}=q_{i} g_{s_{i} w}+\left(q_{i}-1\right) g_{w} .
$$

Thus, in order to compute the product of basis elements we essentially need to be able to compute reduced expressions of products of elements in the underlying Coxeter group. We have described algorithms for computing such reduced expressions for the classical types $A_{n-1}, B_{n}$ and $D_{n}$ in Section 2 . 
Definition. A trace function on $H$ is an $A$-linear map $\lambda: H \rightarrow A$ such that $\lambda\left(h h^{\prime}\right)=$ $\lambda\left(h^{\prime} h\right)$ for all $h, h^{\prime} \in H$.

This definition is in fact valid for any associative algebra over a commutative ground ring. In the case of a group algebra it is clear that every trace function is constant on the conjugacy classes of the underlying group. Since our Iwahori-Hecke algebra $H$ is defined as a deformation of the group algebra of the corresponding Coxeter group $W$, we can hope for similar "deformed" relations between the values of trace functions on $H$. This is indeed true. At first note that the fact that the parameters $q_{i}$ are invertible in $A$ implies that the basis elements $g_{i}$ are invertible in $H$; we have

$$
g_{i}^{-1}=\left(1 / q_{i}\right) g_{i}+\left(1 / q_{i}-1\right) 1_{H} \quad \text { for all } \quad i \in I .
$$

Consequently, every basis element $g_{w}$ (for $w \in W$ ) is invertible in $H$.

In order to understand how conjugation in $W$ translates into $H$, we first note that it is not really conjugation that counts in an algebra. Instead we have to look at the quotient space of $H$ by the commutators. For any $h, h^{\prime} \in H$ we define their commutator by $\left[h, h^{\prime}\right]:=h h^{\prime}-h^{\prime} h$. Let $[H, H] \subseteq H$ be the $A$-subspace of $H$ generated by all commutators. Note that, if two elements $h, h^{\prime}$ are conjugate by some unit in $H$ then $h \equiv h^{\prime} \bmod [H, H]$. Thus, "conjugation" translates to "equivalence modulo commutators".

It is clear that $[H, H]$ lies in the kernel of every trace function on $H$. Conversely, if $\lambda: H \rightarrow A$ is any $A$-linear map which is identically zero on the subspace $[H, H]$ then $\lambda$ is a trace function. We conclude that there is a canonical bijective correspondence between the space of trace functions on $H$ and the dual space of the quotient module $H /[H, H]$. Thus, we have to find out how the images of basis elements $g_{w}$ in $H /[H, H]$ look like. Let us first consider the following two special cases which are in fact the elementary steps in the relations $\longrightarrow$ and $\sim$ defined on $W$ (cf. [GP], Section 1 ).

Let $w, w^{\prime} \in W$ and $i \in I$ such that $w^{\prime}=s_{i} w s_{i}$ and $l\left(w^{\prime}\right)=l(w)$. Then $g_{i}$ conjugates $g_{w}$ to $g_{w^{\prime}}$. More generally, this holds if $w, w^{\prime} \in W$ and $x \in W$ such that $l(w)=l\left(w^{\prime}\right)$, $w^{\prime}=x w x^{-1}$ and $l(x w)=l(x)+l(w)$. For, we then have $g_{w^{\prime}} g_{x}=g_{w^{\prime} x}=g_{x w}=g_{x} g_{w}$, and so $g_{w}$ and $g_{w^{\prime}}$ are conjugate in $H$. (The same conclusion holds if the length condition is $\left.l\left(w x^{-1}\right)=l(w)+l\left(x^{-1}\right).\right)$ Hence, in these cases, we have

$$
g_{w} \equiv g_{w^{\prime}} \quad \bmod \quad[H, H] .
$$

Now let $w, w^{\prime} \in W$ and $i \in I$ such that $w^{\prime}=s_{i} w s_{i}$ and $l\left(w^{\prime}\right)<l(w)$. Then $g_{i}$ conjugates $g_{w}$ to $q_{i} g_{w^{\prime}}+\left(q_{i}-1\right) g_{s_{i} w}$, hence we have

$$
g_{w} \equiv q_{i} g_{w^{\prime}}+\left(q_{i}-1\right) g_{s_{i} w} \quad \bmod \quad[H, H] .
$$

(Note that both $w^{\prime}$ and $s_{i} w$ have length strictly less than $l(w)$.)

Theorem 3.6(a) implies that, for each $w \in W$ there exists some $w^{\prime} \in W$ of minimal length in its conjugacy class such that $w \longrightarrow w^{\prime}$. Applying repeatedly the above relations shows that, modulo $[H, H]$, the basis element $g_{w}$ can be expressed as an $A$-linear combination of basis elements $g_{w^{\prime}}$ where $w^{\prime}$ runs over a set of elements of minimal length in various conjugacy classes. 
Theorem 3.6(b) implies that, if $w, w^{\prime} \in W$ are conjugate and of minimal length in their conjugacy class then $g_{w}$ and $g_{w^{\prime}}$ will be conjugate in $H$. In particular, $g_{w}$ and $g_{w^{\prime}}$ are equal modulo $[H, H]$.

If we take these two statements together we arrive at the following conclusion.

LEMma 4.1. Let $\{C\}$ be the conjugacy classes of $W$. In each class $C$, we choose once and for all a representative $w_{C} \in C$ of minimal length. Then the following holds. For each $w \in W$ there exist constants $f_{w, C} \in A$ such that

$$
g_{w} \equiv \sum_{C} f_{w, C} g_{w_{C}} \quad \bmod \quad[H, H] .
$$

We have $f_{w, C}=0$ unless $l\left(w_{C}\right) \leq l(w)$. The constants $f_{w, C}$ are called class polynomials in $[G P]$; they can be computed recursively using the above rules.

Note that, at this stage, it is conceivable that the class polynomials might not only depend on $w$ and $C$ but also on the sequences of generators which are used to conjugate arbitrary elements in $W$ to those of minimal length in their classes. We will see below that this is not the case. For this purpose, we have to show that the basis elements $g_{w_{C}}$ are linearly independent modulo $[H, H]$. In the case where $A$ is a suitably chosen field, this was already established in [GP]. The general case can be easily deduced from this, using an argument of [GR]. Since this is essential in the existence proof of Markov traces (in fact, of any kind of trace functions) we will give a proof for that general case here.

THEOREM 4.2 (Existence and uniqueness of trace functions). The quotient space $H /[H, H]$ is a free $A$-module. A canonical basis is given by the images (under the natural map $H \rightarrow H /[H, H])$ of the elements $g_{w_{C}}$ where $C$ runs over the conjugacy classes of $W$ and $w_{C}$ is some fixed element of minimal length in $C$.

(The term canonical means that the basis does not depend on the choice of elements $w_{C}$.)

Proof. Lemma 4.1 already implies that the images of the elements $\left\{g_{w_{C}}\right\}$ form a generating set for the $A$-module $H /[H, H]$. It remains to prove their linear independence. For this purpose, it is sufficient to construct a collection of trace functions $\left\{f_{C}\right\}$ on $H$, one for each conjugacy class $C$ of $W$, such that

$$
f_{C}\left(g_{w}\right)=\delta_{C, C^{\prime}} \quad \text { if } \quad w \in C_{\min }^{\prime}
$$

The following arguments are a typical application of the technique of specialization: At first, the desired result is proved in a "generic" situation, for a sufficiently general choice of the ground ring $A$ where the parameters $q_{i}$ are in fact indeterminates. Then the conclusion for any other choice of $A$ and the $q_{i}$ is achieved by "specializing" those indeterminates to the given values.

Step 1: The "generic" situation. Let A be the ring of Laurent polynomials (over the integers) in indeterminates $u_{i}(i \in I)$ such that $u_{i}=u_{j}$ whenever $s_{i}, s_{j}$ are conjugate in $W$. Let $\mathbf{H}$ be the "generic" Iwahori-Hecke algebra associated with $W$ and defined over $\mathbf{A}$ (with parameters $u_{i}$ ). Let $\mathbf{K}$ be an algebraic closure of the field of fractions of $\mathbf{A}$ and $\mathbf{K H}$ the $\mathbf{K}$-algebra obtained from $\mathbf{H}$ by extending scalars from $\mathbf{A}$ to $\mathbf{K}$. Then $\mathbf{K H}$ is a split semisimple $\mathbf{K}$-algebra (see [CR], Corollary 68.12). Hence every trace function on $\mathbf{K H}$ is a linear combination of the characters of the irreducible representations of $\mathbf{K H}$. 
Moreover, by Tits' Deformation Theorem (see [CR], Theorem 68.21), the algebra $\mathbf{K H}$ is in fact isomorphic to the group algebra of $W$ over $\mathbf{K}$. It follows that the number of irreducible characters of $\mathbf{K H}$ equals the number of conjugacy classes of $W$, and that the (square) matrix of values $\left(\chi\left(\mathbf{g}_{w_{C}}\right)\right)$, where $\chi$ runs over the irreducible characters of KH and $C$ runs over the conjugacy classes of $W$, is invertible. This immediately implies that the basis elements $\mathbf{g}_{w_{C}}$ are linearly independent modulo $[\mathbf{K H}, \mathbf{K H}]$, and hence the class polynomials $\mathbf{f}_{w, C}$ of Lemma 4.1 are uniquely determined. The definition of the class polynomials shows that $\mathbf{f}_{w, C}=\delta_{C, C^{\prime}}$ for $w \in C_{\text {min }}^{\prime}$.

The following arguments are taken from [GR], Section 5.1. For each class $C$, we can define a linear map $\mathbf{f}_{C}: \mathbf{K H} \rightarrow \mathbf{K}$ by

$$
\mathbf{f}_{C}\left(\mathbf{g}_{w}\right):=\mathbf{f}_{w, C} \text { for all } w \in W .
$$

We check that $\mathbf{f}_{C}$ is a trace function on $\mathbf{K H}$. Indeed, for each $w \in W$, we have equations

$$
\chi\left(\mathbf{g}_{w}\right)=\sum_{C} \mathbf{f}_{w, C} \chi\left(\mathbf{g}_{w_{C}}\right) \text { for all irreducible characters } \chi .
$$

We can invert these equations and hence obtain expressions of the functions $\mathbf{f}_{C}$ as linear combinations of the irreducible characters of $\mathbf{K H}$. Hence the functions $\mathbf{f}_{C}$ must be trace functions themselves.

Finally, we note that the class polynomials of $\mathbf{H}$ and $\mathbf{K H}$ clearly are the same, and that they lie in $\mathbf{A}$. Hence the functions $\mathbf{f}_{C}$ restrict to trace functions on $\mathbf{H}$ with values in $\mathbf{A}$. This collection of trace functions on $\mathbf{H}$ has the required properties.

Step 2: "Specialization". Given any other choice for $A$ and the parameters $\left\{q_{i}\right\}$, we have a unique ring homomorphism $\varphi: \mathbf{A} \rightarrow A$ such that $\varphi\left(u_{i}\right)=q_{i}$ for all $i$. Then the Iwahori-Hecke algebra $H$ (over $A$ and with parameters $\left\{q_{i}\right\}$ ) can also be described as the tensor product $A \otimes_{\mathbf{A}} \mathbf{H}$ where $A$ becomes an $\mathbf{A}$-module via $\varphi$. This operation of extending scalars from $\mathbf{A}$ to $A$ also induces a canonical map from the space of trace functions on $\mathbf{H}$ to the space of trace functions on $H$. (In more concrete terms, the following happens. Let $\mathbf{a}\left(w, w^{\prime}, w^{\prime \prime}\right) \in \mathbf{A}$ be the structure constants for the multiplication of basis elements in the generic algebra $\mathbf{H}$, that is, we have

$$
\mathbf{g}_{w} \cdot \mathbf{g}_{w^{\prime}}=\sum_{w^{\prime \prime} \in W} \mathbf{a}\left(w, w^{\prime}, w^{\prime \prime}\right) \mathbf{g}_{w^{\prime \prime}} \quad \text { for } \quad w, w^{\prime} \in W .
$$

Then the elements $\varphi\left(\mathbf{a}\left(w, w^{\prime}, w^{\prime \prime}\right)\right) \in A$ are the structure constants for the multiplication of basis elements in the algebra $H$ over $A$. If $\mathbf{f}: \mathbf{H} \rightarrow \mathbf{A}$ is a trace function on $\mathbf{H}$ then the map $\mathbf{f}_{\varphi}: H \rightarrow A$ defined by $g_{w} \mapsto \varphi\left(\mathbf{f}\left(\mathbf{g}_{w}\right)\right)$, is a trace function on $H$.) The images of the functions $\mathbf{f}_{C}$ then have the required properties. This completes the proof of the Theorem.

Using the duality between trace functions and the quotient space modulo the commutators, we can immediately translate the last two results to trace functions.

Lemma 4.1 implies that every trace function on $H$ is uniquely determined by its values on the basis elements $g_{w_{C}}$, where $C$ runs over the conjugacy classes of $W$.

Theorem 4.2 implies that, conversely, if $\left\{a_{C}\right\}$ is a collection of elements in $A$, one for each conjugacy class $C$ of $W$, then there exists a unique trace function $\lambda$ on $H$ such that $\lambda\left(g_{w}\right)=a_{C}$ if the element $w \in W$ has minimal length in the class $C$. 
5. Definition and basic properties of Markov traces. Let again $X_{n}$ (for $n \geq 1$ ) be one of the classical types $A_{n-1}, B_{n}$ or $D_{n}$, and $W\left(X_{n}\right)$ the corresponding finite Coxeter group. We have natural embeddings $W\left(X_{1}\right) \subset W\left(X_{2}\right) \subset \ldots$, and we let

$$
W_{\infty}:=\bigcup_{n \geq 1} W\left(X_{n}\right)
$$

Let $H\left(X_{n}\right)$ be the associated Iwahori-Hecke algebra defined over a commutative ring $A$ as in Section 4. Since all generators $s_{i}$ are conjugate, the corresponding parameters $q_{i}$ must be equal, and we denote them by $q$. In type $B_{n}$, the parameter corresponding to the generator $t$ will be denoted by $Q$. In type $D_{n}$, the parameter corresponding to the generator $u$ also equals $q$ (since $u$ is conjugate to $s_{1}$, as soon as $n \geq 3$ ). We also have natural embeddings $H\left(X_{1}\right) \subset H\left(X_{2}\right) \subset \ldots$, and we let

$$
H_{\infty}:=\bigcup_{n \geq 1} H\left(X_{n}\right)
$$

The algebra $H_{\infty}$ is a free $A$-algebra of infinite rank, generated by the elements $g_{1}, g_{2}, \ldots$ (corresponding to the generators $s_{1}, s_{2}, \ldots$ ), and an additional generator which we denote by $T$ in type $B_{n}$, and by $U$ in type $D_{n}$.

Definition (cf. [Bi], p. 264) Let $z \in A$ and $\tau: H_{\infty} \rightarrow A$ be $A$-linear. Then $\tau$ is called a Markov trace (with parameter $z$ ) if the following conditions are satisfied.

(1) $\tau$ is a trace function.

(2) $\tau(1)=1$ (normalization).

(3) $\tau\left(h g_{n}\right)=z \tau(h)$ for all $n \geq 1$ and $h \in H\left(X_{n}\right)$ (Markov property).

We note that all generators $g_{i}$ (for $i=1,2, \ldots$ ) are conjugate in $H_{\infty}$. In particular, any trace function on $H_{\infty}$ must have the same value on these elements. This explains why the parameter $z$ is independent of $n$ in rule (3) of this definition.

The crucial step in the classification of Markov traces is the observation that the values of such a trace on basis elements of $H_{\infty}$ corresponding to elements in signed block form in $W_{\infty}$ only depend on the number of positive and negative blocks if we replace some generators $g_{i}$ by their inverses. For this purpose, recall the definition of the sets $\mathcal{D}\left(X_{n}\right)$ and the double coset representatives $t_{n-1}$ (in type $B_{n}$ ) and $u_{n-1}$ (in type $D_{n}$ ) from Section 3. We define corresponding elements in the Iwahori-Hecke algebras as follows.

$$
T_{n-1}^{\prime}:=g_{n-1} \cdots g_{1} T g_{1}^{-1} \cdots g_{n-1}^{-1} \text { for all } n \geq 1 \text { in type } B \text {, }
$$

and

$$
U_{n-1}^{\prime}:=g_{n-1} \cdots g_{2} U g_{1}^{-1} g_{2}^{-1} \cdots g_{n-1}^{-1} \text { for all } n \geq 2 \text { in type } D
$$

The braid relations in $H\left(X_{n}\right)$ imply that the generators $g_{1}, \ldots, g_{n-2}$ commute with $T_{n-1}^{\prime}$ in type $B_{n}$, and that the generators $g_{2}, \ldots, g_{n-2}$ commute with $U_{n-1}^{\prime}$ in type $D_{n}$, while $U U_{n-1}^{\prime}=U_{n-1}^{\prime} g_{1}$ but $g_{1} U_{n-1}^{\prime} \neq U_{n-1}^{\prime} U$ for all $n \geq 3$ !

We use these elements to define the analogues of the sets $\mathcal{D}\left(X_{n}\right)$ as subsets of $H_{\infty}$. If $n=1$, we let $\mathcal{D}^{\prime}\left(X_{1}\right):=\left\{g_{w} \mid w \in W\left(X_{1}\right)\right\}$. For $n \geq 2$ we give the definition case by case. 
Type $A_{n-1}: \quad \mathcal{D}^{\prime}\left(X_{n}\right):=\left\{1, g_{n-1}\right\}$.

Type $B_{n}: \mathcal{D}^{\prime}\left(X_{n}\right):=\left\{1, g_{n-1}, T_{n-1}^{\prime}\right\}$

Type $D_{n} \quad: \quad \mathcal{D}^{\prime}\left(X_{n}\right):=\left\{1, g_{n-1}, U_{n-1}^{\prime}\right\}$ for $n \geq 3$ and $\mathcal{D}^{\prime}\left(X_{2}\right):=\left\{1, g_{1}, U, U_{1}^{\prime}\right\}$.

Now recall from Section 4 that a trace function on an Iwahori-Hecke algebra is uniquely determined by its values on basis elements corresponding to representatives of minimal length in the various conjugacy classes of the underlying Coxeter group. Also recall from Section 3 that representatives of minimal length in the classes of Coxeter groups of classical types are of the form $d_{1} \cdots d_{n}$, where $d_{i}$ is a distinguished double coset representative of $W\left(X_{i}\right)$ with respect to $W\left(X_{i-1}\right)$. If we are also willing to consider elements in signed block form which have not necessarily minimal length in their class we arrive at the following result (which already appeared in [GL], Proposition 3.4 and Corollary 3.5, for type $B$ ). It translates Proposition 3.2 from the level of Coxeter groups to the level of Iwahori-Hecke algebras, taking into account the modified sets $\mathcal{D}^{\prime}\left(X_{n}\right)$.

Lemma 5.1. Let $h \in H\left(X_{n}\right)$ for some $n \geq 1$. Then there exists a function $a_{h}$ : $\mathcal{D}^{\prime}\left(X_{1}\right) \times \cdots \times \mathcal{D}^{\prime}\left(X_{n}\right) \rightarrow A$ such that

$$
\lambda(h)=\sum_{\left(d_{1}^{\prime}, \cdots, d_{n}^{\prime}\right)} a_{h}\left(d_{1}^{\prime}, \ldots, d_{n}^{\prime}\right) \lambda\left(d_{1}^{\prime} \cdots d_{n}^{\prime}\right)
$$

and

$$
\lambda\left(h g_{n}\right)=\sum_{\left(d_{1}^{\prime}, \cdots, d_{n}^{\prime}\right)} a_{h}\left(d_{1}^{\prime}, \ldots, d_{n}^{\prime}\right) \lambda\left(d_{1}^{\prime} \cdots d_{n}^{\prime} g_{n}\right)
$$

for all trace functions $\lambda: H \rightarrow A$ (where $d_{i}^{\prime} \in \mathcal{D}^{\prime}\left(X_{i}\right)$ for all $\left.i\right)$.

Proof. We may clearly assume that $h=g_{w}$ for some $w \in W\left(X_{n}\right)$. We proceed by induction on $l(w)$. If $l(w)=0$ or $l(w)=1$, there is nothing to prove. Now let $l(w) \geq 2$ (and hence $n \geq 2$ ). Recall from Proposition 3.2 that for each $w \in W\left(X_{n}\right)$ there exists a sequence of generators in $W\left(X_{n-1}\right)$ which conjugates $w$ step by step to an element of the form $d_{1} \cdots d_{n}$ (with $d_{i} \in \mathcal{D}\left(X_{i}\right)$ for all $i$ ) in such a way that the length either remains the same or decreases at each step. (Note that we don't necessarily reach representatives of minimal length in the classes of $W\left(X_{n}\right)$.) Since the generators in $W\left(X_{n-1}\right)$ commute with $s_{n}$ we thus have

$$
w \longrightarrow d_{1} \cdots d_{n} \text { and } w s_{n} \longrightarrow d_{1} \cdots d_{n} s_{n}
$$

where in both cases we can use the same sequence of generators.

If we apply the analogous conjugations to the basis elements in $H\left(X_{n}\right)$ and use the relations in the beginning of Section 4, we find the following. For each $w \in W\left(X_{n}\right)$ there exists a function $b_{w}: \mathcal{D}\left(X_{1}\right) \times \cdots \times \mathcal{D}\left(X_{n}\right) \rightarrow A$ such that

$$
g_{w} \equiv \sum_{\left(d_{1}, \ldots, d_{n}\right)} b_{w}\left(d_{1}, \ldots, d_{n}\right) g_{d_{1}} \cdots g_{d_{n}} \bmod \quad\left[H\left(X_{n}\right), H\left(X_{n}\right)\right]
$$

and

$$
g_{w} g_{n} \equiv \sum_{\left(d_{1}, \ldots, d_{n}\right)} b_{w}\left(d_{1}, \ldots, d_{n}\right) g_{d_{1}} \cdots g_{d_{n}} g_{n} \quad \bmod \quad\left[H\left(X_{n}\right), H\left(X_{n}\right)\right]
$$


Now we have to replace the basis elements corresponding to the elements $d_{i}$ by the modified elements $d_{i}^{\prime}$. If $d_{i}=g_{i-1}$ there is nothing to be changed. So let us assume that $d_{i}=t_{i-1}$ (in type $B_{n}$ ) or $d_{i}=u_{i-1}$ (in type $D_{n}$ ). Consider the corresponding product of the generators in $H\left(X_{i}\right)$. Replacing the appropriate generators $g_{j}$ by their inverses and using the inversion formula for $g_{j}$ shows that

$$
g_{d_{i}}=q^{l\left(d_{i}\right)} d_{i}^{\prime}+A \text {-linear combination of } g_{v} \text { with } v \in W\left(X_{i}\right) \text { and } l(v)<l\left(d_{i}\right)
$$

where $d_{i}^{\prime}$ is the element in $\mathcal{D}^{\prime}\left(X_{i}\right)$ analogous to $d_{i}$. We insert these expressions in the above relations and conclude that

$$
g_{w} \equiv \tilde{h}+\sum_{\left(d_{1}, \ldots, d_{n}\right)} b_{w}\left(d_{1}, \ldots, d_{n}\right) d_{1}^{\prime} \cdots d_{n}^{\prime} \quad \bmod \quad\left[H\left(X_{n}\right), H\left(X_{n}\right)\right]
$$

and

$$
g_{w} g_{n} \equiv \tilde{h} g_{n}+\sum_{\left(d_{1}, \ldots, d_{n}\right)} b_{w}\left(d_{1}, \ldots, d_{n}\right) d_{1}^{\prime} \cdots d_{n}^{\prime} g_{n} \quad \bmod \quad\left[H\left(X_{n}\right), H\left(X_{n}\right)\right]
$$

where $\tilde{h} \in H\left(X_{n}\right)$ is a linear combination of basis elements corresponding to elements in $W\left(X_{n}\right)$ of strictly smaller length than $w$. We can then apply induction to complete the proof.

The above Lemma allows us to reduce the computation of the value of any trace function on $H_{\infty}$ to the computation of the values on elements of the form $d_{1}^{\prime} \cdots d_{n}^{\prime}$, with $d_{i}^{\prime} \in \mathcal{D}^{\prime}\left(X_{i}\right)$ for all $i$. The next result is the key to the evaluation of Markov traces on such elements. For type $B$, this already appeared in [GL], Lemma 4.2. It shows that we can always apply a Markov type rule to factors $g_{n}$, and shift the remaining factors $T_{n}^{\prime}$ or $U_{n}^{\prime}$ to the left as far as possible.

Lemma 5.2. Let $\tau$ be a Markov trace on $H_{\infty}$ with parameter $z$. To abbreviate notation, we let $Y_{i}$ denote the element $T_{i}^{\prime}$ (in type $B$ ) respectively $U_{i}^{\prime}$ (in type $\left.D\right)$.

(a) Let $n \geq 1$ in type $B$ and $n \geq 2$ in type $D$. If $h \in H\left(X_{n}\right)$ and $m \geq 0$, then

$$
\tau\left(h g_{n} Y_{n+1} \cdots Y_{n+m}\right)=z \tau\left(h Y_{n} \cdots Y_{n+m-1}\right) .
$$

(b) Let $n \geq 1$. If $h \in H\left(X_{n}\right)$ and $m \geq 0$, then

$$
\tau\left(h Y_{n+1} \cdots Y_{n+m}\right)=\tau\left(h Y_{n} \cdots Y_{n+m-1}\right) .
$$

Proof. To prove these relations we will proceed by induction on $m$. If $m=0$ then there is nothing to prove in (b), while in (a) we can apply directly rule (3) in the definition of Markov traces. Now let us assume that $m>0$. Let $x=g_{n}$ in (a) and $x=1$ in (b). We have to evaluate the expression

$$
\tau\left(h x Y_{n+1} \cdots Y_{n+m}\right) .
$$

Since $n \geq 1$ we can write $Y_{n+1}=g_{n+1} Y_{n} g_{n+1}^{-1}$. The braid relations in $H$ imply that $g_{n+1}^{-1}$ commutes with $Y_{n+2}, \ldots, Y_{n+m}$. Since $\tau$ is a trace our expression is equal to

$$
\tau\left(g_{n+1}^{-1} h x g_{n+1} Y_{n} Y_{n+2} \cdots Y_{n+m}\right) .
$$


Now $h$ lies in $H\left(X_{n}\right)$, that is, $h$ only involves the generators $t, g_{1}, \ldots, g_{n-1}$. It follows that $h$ commutes with $g_{n+1}^{-1}$. Hence, if $x=1$ we find that our expression equals

$$
\tau\left(h Y_{n} Y_{n+2} \cdots Y_{n+m}\right) .
$$

We can apply induction with $h^{\prime}:=h Y_{n}$, and we are done.

We let $x=g_{n}$ from now on. Using the braid relation $g_{n+1}^{-1} g_{n} g_{n+1}=g_{n} g_{n+1} g_{n}^{-1}$, the above expression can be rewritten as

$$
\tau\left(h g_{n} g_{n+1} g_{n}^{-1} Y_{n} Y_{n+2} \cdots Y_{n+m}\right) .
$$

If we are in type $B$ with any $n$, respectively in type $D$ with $n \geq 2$, we can write $Y_{n}=$ $g_{n} Y_{n-1} g_{n}^{-1}$. Then the left hand term $g_{n}$ will cancel and then $g_{n+1}$ commutes with $Y_{n-1}$. Now our expression reads

$$
\tau\left(h g_{n} Y_{n-1} g_{n+1} g_{n}^{-1} Y_{n+2} \cdots Y_{n+m}\right) .
$$

The element $g_{n}^{-1}$ commutes with all terms to the right of it. Hence our expression equals

$$
\tau\left(g_{n}^{-1} h g_{n} Y_{n-1} g_{n+1} Y_{n+2} \cdots Y_{n+m}\right) \text {. }
$$

We write $h^{\prime}:=g_{n}^{-1} h g_{n} Y_{n-1}$ and observe that this element lies in $H_{n+1}$. So we can apply the induction and obtain that

$$
\tau\left(h g_{n} Y_{n+1} \cdots Y_{n+m}\right)=\tau\left(h^{\prime} g_{n+1} Y_{n+2} \cdots Y_{n+m}\right)=z \tau\left(h^{\prime} Y_{n+1} \cdots Y_{n+m-1}\right) .
$$

We insert the expression for $h^{\prime}$ again, note that $g_{n}^{-1}$ commutes with $Y_{n+1} \cdots Y_{n+m-1}$, and conclude that

$$
\tau\left(h^{\prime} Y_{n+1} \cdots Y_{n+m}\right)=\tau\left(h g_{n} Y_{n-1} g_{n}^{-1} Y_{n+1} \cdots Y_{n+m}\right)=z \tau\left(h Y_{n} Y_{n+1} \cdots Y_{n+m-1}\right) .
$$

Putting things together we see that this completes the proof.

5.3. Markov traces for type $A$. Let $\tau$ be a Markov trace with parameter $z$, and let $d_{i}^{\prime} \in \mathcal{D}^{\prime}\left(A_{i-1}\right)$ for $i=1, \ldots, n$. Then each $d_{i}^{\prime}$ either equals 1 or $g_{i-1}$, hence the Markov property directly implies that

$$
\tau\left(d_{1}^{\prime} \cdots d_{n}^{\prime}\right)=z^{a}
$$

where $a$ is the number of factors equal to $g_{i-1}$. Thus, $\tau$ is uniquely determined by its parameter $z$.

To prove the existence of a Markov trace with any given parameter $z \in A$, we refer to [Jo], Theorem 5.1, or [GP], (4.1). In the framework of the theory developed here, the argument is as follows. We define a trace that has the Markov property on the elements $d_{1}^{\prime} \cdots d_{n}^{\prime}$ corresponding to elements of minimal length in the conjugacy classes of $W_{\infty}$, and we have to show that the Markov property then holds for all elements of $H_{\infty}$.

In particular for type $A$ we have, by (3.3), that each element of the form $d_{1} \cdots d_{n}$, with $n \geq 1$ and $d_{i} \in \mathcal{D}\left(A_{i-1}\right)$ for all $i$, has minimal length in its class in $W\left(A_{n-1}\right)$. Note that the corresponding elements $d_{i}^{\prime} \in \mathcal{D}^{\prime}\left(A_{i-1}\right)$ are just $g_{d_{i}}$. By Theorem 4.2 we can define a trace function $\tau_{n}: H\left(A_{n-1}\right) \rightarrow A$ by specifying its values on elements of the form $d_{1}^{\prime} \cdots d_{n}^{\prime}$, and the Markov property forces us to let $\tau\left(d_{1}^{\prime} \cdots d_{n}^{\prime}\right)=z^{a}$, with $a$ as above. These traces are compatible with the embeddings $H\left(A_{1}\right) \subset H\left(A_{2}\right) \subset \ldots$, hence they define a unique trace function on the infinite union of these algebras. It remains to 
show that the Markov property holds for all elements of $H_{\infty}$. This follows by combining the two equations in Lemma 5.1.

5.4. Markov traces for type $B$. Let $\tau$ be a Markov trace with parameter $z$, and let $d_{i}^{\prime} \in \mathcal{D}^{\prime}\left(B_{i}\right)$ for $i=1, \ldots, n$. Then each $d_{i}^{\prime}$ either equals 1 or $g_{i-1}$ or $T_{i-1}^{\prime}$, hence Lemma 5.2 implies that

$$
\tau\left(d_{1}^{\prime} \cdots d_{n}^{\prime}\right)=z^{a} \tau\left(T_{0}^{\prime} \cdots T_{b-1}^{\prime}\right),
$$

where $a$ is the number of factors $d_{i}^{\prime}$ which are equal to $g_{i-1}$ and $b$ is the number of factors $d_{i}^{\prime}$ which are equal to $T_{i-1}^{\prime}$. Thus, $\tau$ is uniquely determined by its parameter $z$ and the values on the elements in the set

$$
\left\{T_{0}^{\prime} \cdots T_{k-1}^{\prime} \mid k=1,2, \ldots\right\} .
$$

Conversely, given $z, y_{1}, y_{2}, \ldots \in A$ there does exist a Markov trace $\tau$ with parameter $z$ and such that $\tau\left(T_{0}^{\prime} T_{1}^{\prime} \cdots T_{k-1}^{\prime}\right)=y_{k}$ for all $k \geq 1$. For the details of this existence result, we refer to [GL], Theorem 4.3. The argument is very roughly analogous to type $A$ but complications arise from the fact that one also has to consider conjugates of $T$ where the conjugating element is of the form $g_{1}^{ \pm 1} \cdots g_{i}^{ \pm 1}$, with any choice of signs.

6. Markov traces for type $D$. The aim of this section is to classify Markov traces for Iwahori-Hecke algebras of type $D$. The best way to achieve this seems to be by embedding these algebras into those for type $B$, and then to use the known results for type $B$. To fix notation we let $W_{n}$ (for $n \geq 2$ ) denote the Coxeter group of type $B_{n}$, with generators $\left\{t, s_{1}, \ldots, s_{n-1}\right\}$ and $W_{n}^{\prime} \subseteq W_{n}$ the Coxeter group of type $D_{n}$, with generators $\left\{u=t s_{1} t, s_{1}, \ldots, s_{n-1}\right\}$ (see Section 2.3). We let

$$
W_{\infty}^{\prime}:=\bigcup_{n \geq 2} W_{n}^{\prime} \subset W_{\infty}:=\bigcup_{n \geq 2} W_{n} .
$$

We denote the Iwahori-Hecke algebras of type $B_{n}$ and $D_{n}$ by $H_{n}$ and $H_{n}^{\prime}$, respectively. In order to have an embedding of $H_{n}^{\prime}$ into $H_{n}$, we have to set the parameter $Q$ equal to 1 . Indeed, in this case, we have $T^{2}=1$ in $H_{n}$ and can compute that

$$
U^{2}=\left(T g_{1} T\right)^{2}=T g_{1}^{2} T=q \cdot 1_{H}+(q-1) T g_{1} T=q \cdot 1_{H}+(q-1) U .
$$

Furthermore, we have a decomposition of $A$-modules $H_{n}=H_{n}^{\prime} \oplus H_{n}^{\prime} T$ where $H_{n}^{\prime} T=T H_{n}^{\prime}$. Thus, $H_{n}^{\prime}$ is the subalgebra of $H_{n}$ generated by the elements $U=T g_{1} T, g_{1}, \ldots, g_{n-1}$. We let

$$
H_{\infty}^{\prime}:=\bigcup_{n \geq 2} H_{n}^{\prime} \quad \subset \quad H_{\infty}:=\bigcup_{n \geq 2} H_{n}
$$

and consider the restriction of trace functions from $H_{\infty}$ to $H_{\infty}^{\prime}$. It is clear that the restriction of a Markov trace on $H_{\infty}$ is a Markov trace on $H_{\infty}^{\prime}$ (and both have the same parameter). Our main result will show that every Markov trace on $H_{\infty}^{\prime}$ can be obtained in this way.

At first we will establish a uniqueness result for Markov traces on $H_{\infty}^{\prime}$, similarly to that for $H_{\infty}$. Recall that we have $\mathcal{D}^{\prime}\left(D_{2}\right)=\left\{1, g_{1}, U, U_{1}^{\prime}\right\}$ and $\mathcal{D}^{\prime}\left(D_{i}\right)=\left\{1, g_{i-1}, U_{i-1}^{\prime}\right\}$ for $i \geq 3$, where

$$
U_{i}^{\prime}=g_{i} \cdots g_{2} U g_{1}^{-1} g_{2}^{-1} \cdots g_{i}^{-1} \quad \text { for all } \quad i \geq 1
$$


The main difference to type $B$ lies in the set $\mathcal{D}^{\prime}\left(D_{2}\right)$ which does not just contain the elements $g_{1}$ and $U_{1}^{\prime}$ (as would be analogous to type $B$ ) but also the extra generator $U$. The main complication arising from this is that $g_{1}$ respectively $U$ do not commute with $U_{i}^{\prime}$ for $i \geq 2$. On the level of $W_{n}^{\prime}$ we have $u u_{i}=u_{i} s_{1}$ and $u_{i} u=s_{1} u_{i}$, but on the level of $H_{n}^{\prime}$ we only have $U U_{i}^{\prime}=U_{i}^{\prime} g_{1}$ but $U_{i}^{\prime} U \neq g_{1} U_{i}^{\prime}$ for all $i \geq 2$. Nevertheless, we have the following relations:

$$
\begin{aligned}
& U^{ \pm 1} U_{i}^{\prime}=U_{i}^{\prime} g_{1}^{ \pm 1}+A \text {-linear combination of } g_{w} \text { with } w \in W_{n}^{\prime} \text { and } l(w)<l\left(u u_{i}\right), \\
& g_{1}^{ \pm 1} U_{i}^{\prime}=U_{i}^{\prime} U^{ \pm 1}+A \text {-linear combination of } g_{w} \text { with } w \in W_{n}^{\prime} \text { and } l(w)<l\left(u u_{i}\right) .
\end{aligned}
$$

(Indeed, we know that $u u_{i}=u_{i} s_{1}$ and $s_{1} u_{i}=u_{i} u$. So we also have $U U_{i}=U_{i} g_{1}$ and $g_{1} U_{i}=U_{i} U$ where $U_{i}=g_{i} \cdots g_{2} U g_{1} g_{2} \cdots g_{i}$. The above formulas then follow by inserting the expressions $g_{j}=q g_{j}^{-1}+(q-1) 1_{H}$ or $U=q U^{-1}+(q-1) 1_{H}$ at the appropriate places, and expanding.)

We will argue carefully by induction on the length of elements, and then the above two rules will be sufficient.

Proposition 6.1 (Uniqueness of Markov traces for type D). Let $z \in A$ and $\tau: H_{\infty}^{\prime} \rightarrow$ $A$ be a Markov trace with parameter $z$. Then $\tau$ is uniquely determined by its values on the elements in the set

$$
\left\{U_{1}^{\prime} \cdots U_{2 k-1}^{\prime} \mid k=1,2, \ldots\right\} .
$$

Proof. For each $N \geq 0$ let $H^{\prime}(\leq N)$ be the $A$-subspace of $H^{\prime}$ generated by all elements $g_{w}$ with $w \in W_{n}^{\prime}$ for some $n \geq 2$ and $l(w) \leq N$. The subspace $H^{\prime}(<N)$ is defined in the analogous way. We claim that, for all $N$, the values of $\tau$ on elements in $H^{\prime}(\leq N)$ are determined by the values on elements of the form $U_{1}^{\prime} \cdots U_{2 k-1}^{\prime}$, for $k=1,2, \ldots$ If $N=0$ there is nothing to prove. Now assume that our claim holds for $N \geq 0$ and that $w \in W_{n}^{\prime}$ for some $n$ such that $l(w)=N+1$. Since the $W_{n}$ form an increasing chain we may assume that $n$ is large enough. (Actually, we shall use that $n \geq 3$.) We must show that $\tau\left(g_{w}\right)$ is determined as required.

By Lemma 4.1, $\tau\left(g_{w}\right)$ can be expressed as a linear combination of values $\tau\left(g_{w_{C}}\right)$ where $C$ runs over a set of conjugacy classes of $W_{n}^{\prime}$ such that $l\left(w_{C}\right) \leq l(w)$. Let us fix such a class $C$, labelled by the pair of partitions $(\alpha, \beta)$ say. The discussion in (3.5) shows that two different types of classes can occur.

Type I. The elements $w_{C}$ and $t w_{C} t$ are not conjugate in $W_{n}^{\prime}$. Then $w_{C}$ must have a very special form: Up to possibly interchanging the roles of $w_{C}$ and $t w_{C} t$, we must have $w_{C}=s_{1} s_{i_{1}} \cdots s_{i_{r}}$ and hence $t w_{C} t=u s_{i_{1}} \cdots s_{i_{r}}$, for some $2 \leq i_{1}<\cdots<i_{r}$. Using the Markov property, we can therefore compute that

$$
\tau\left(g_{w_{C}}\right)=\tau\left(g_{1} g_{i_{1}} \cdots g_{i_{r}}\right)=\tau\left(g_{1}\right) z^{r} \quad \text { and } \quad \tau\left(g_{t w_{C} t}\right)=\tau\left(U g_{i_{1}} \cdots g_{i_{r}}\right)=\tau(U) z^{r} .
$$

Now we observe that $u$ and $s_{1}$ are two generators of $W_{n}^{\prime}$, and that all generators are conjugate (since $n \geq 3$ ). Clearly, they are of minimal length in their conjugacy class. Using Theorem 3.6(b) and the Markov property, we conclude that $\tau(U)=\tau\left(g_{1}\right)=z$. Hence, the values of $\tau$ on $g_{w_{C}}$ and $g_{t w_{C} t}$ are already determined (they are equal to $z^{r+1}$ ).

Type II. The elements $w_{C}$ and $t w_{C} t$ are conjugate in $W_{n}^{\prime}$. If the partition $\alpha$ is empty, then we can assume that $w_{C}$ is a product of generators $s_{i}$, hence the Markov property 
alone determines the value of $\tau$ on $g_{w}$. From now on, let us assume that $\alpha$ is not empty. We can assume that $w_{C}=d_{1} \cdots d_{n}$ with $d_{i} \in \mathcal{D}\left(D_{i}\right)$ for all $i$. As in the proof of Lemma 5.1 we see that, up to a power of $q$, the element $g_{w_{C}}=g_{d_{1} \cdots d_{n}}$ is congruent to $d_{1}^{\prime} \cdots d_{n}^{\prime}$ modulo $H^{\prime}\left(<l\left(w_{C}\right)\right) \subseteq H^{\prime}(\leq N)$ where $d_{i}^{\prime} \in \mathcal{D}^{\prime}\left(D_{i}\right)$ is the element of the analogous form as $d_{i}$. By induction, it is therefore sufficient to show that the value of $\tau$ on $d_{1}^{\prime} \cdots d_{n}^{\prime}$ is determined as required.

Recall from (3.5) that $w_{C}=w(\alpha, \beta)^{\prime}$ where $\alpha$ has an even number of parts and elements of the form $u_{i} s_{i+1} \cdots s_{i+d-1}$ are considered as negative blocks (with the convention that $u_{0}=1$ ). Hence the total number of "proper" factors $u_{i} \neq 1$ in this expression for $w_{C}$ is odd!

If the first negative block has length 1 it just consists of $u_{0}=1$ hence in our expression $d_{1}^{\prime} \cdots d_{n}^{\prime}$ we have $d_{1}^{\prime}=1, d_{2}^{\prime}=U_{1}^{\prime}$. We can directly apply Lemma 5.2 and conclude that

$$
\tau\left(d_{1}^{\prime} \cdots d_{n}^{\prime}\right)=z^{a} \tau\left(U_{1}^{\prime} \cdots U_{2 b-1}^{\prime}\right)
$$

where $a$ is the number of factors $d_{i}^{\prime}$ equal to $g_{i-1}$ and $2 b$ is the number of parts of $\alpha$.

If the first negative block has length $>1$ it consists of a product of factors $s_{1} \cdots s_{m-1}$ for some $m \geq 2$ hence in our expression $d_{1}^{\prime} \cdots d_{n}^{\prime}$ we have $d_{1}^{\prime}=1, d_{2}^{\prime}=g_{1}, \ldots, d_{m}^{\prime}=g_{m-1}$. Using Lemma 5.2, we conclude that

$$
\tau\left(d_{1}^{\prime} \cdots d_{n}^{\prime}\right)=z^{a} \tau\left(g_{1} U_{2}^{\prime} \cdots U_{2 b}^{\prime}\right)
$$

where $a$ is the number of factors $d_{i}^{\prime}$ which are equal to $g_{i-1}$ and $2 b$ is the number of parts of $\alpha$. We write $U_{2}^{\prime}=g_{2} U g_{1}^{-1} g_{2}^{-1}$ and obtain that

$$
\tau\left(g_{1} U_{2}^{\prime} \cdots U_{2 b}^{\prime}\right)=\tau\left(g_{1} g_{2} U g_{1}^{-1} g_{2}^{-1} U_{3}^{\prime} \cdots U_{2 b}^{\prime}\right)=\tau\left(g_{2}^{-1} g_{1} g_{2} U g_{1}^{-1} U_{3}^{\prime} \cdots U_{2 b}^{\prime}\right),
$$

since $g_{2}$ and hence also $g_{2}^{-1}$ commutes with $U_{i}^{\prime}$ for $i \geq 3$. Now we use the braid relation $g_{2}^{-1} g_{1} g_{2}=g_{1} g_{2} g_{1}^{-1}$ and rewrite the above expression as $\tau\left(g_{1} g_{2} U g_{1}^{-2} U_{3}^{\prime} \cdots U_{2 b}^{\prime}\right)$. We apply the above commutation rules for $g_{1}^{-2}, U$ with $U_{3}^{\prime} \cdots U_{2 b}^{\prime}$ and conclude that

$$
g_{1} g_{2} U g_{1}^{-2} U_{3}^{\prime} \cdots U_{2 b}^{\prime} \equiv U g_{1}^{-2} g_{1} g_{2} U_{3}^{\prime} \cdots U_{2 b}^{\prime}=U_{1}^{\prime} g_{2} U_{3}^{\prime} \cdots U_{2 b}^{\prime} \quad \bmod \quad H(\leq N)^{\prime} .
$$

Now Lemma 5.2 implies that

$$
\tau\left(U_{1}^{\prime} g_{2} U_{3}^{\prime} \cdots U_{2 b}^{\prime}\right)=z \tau\left(U_{1}^{\prime} U_{2}^{\prime} \cdots U_{2 b-1}^{\prime}\right) .
$$

This completes the proof.

Theorem 6.2 (Classification of Markov traces for type $D)$. Let $z, y_{2}, y_{4}, \ldots \in A$. Then there exists a unique Markov trace $\tau$ on $H_{\infty}^{\prime}$ with parameter $z$ such that

$$
\tau\left(U_{1}^{\prime} \cdots U_{2 k-1}^{\prime}\right)=y_{2 k} \quad \text { for all } k \geq 1 \text {. }
$$

Every Markov trace on $H_{\infty}^{\prime}$ is the restriction of a Markov trace on $H_{\infty}$.

P r o o f. Uniqueness was already proved in Proposition 6.1. In order to prove existence, choose any elements $y_{1}, y_{3}, \ldots \in A$, in addition to the given elements $z, y_{2}, y_{4}, \ldots \in A$. By the classification of Markov traces for type $B$ (see (5.4) and [GL], Theorem 4.3), there exists a (unique) Markov trace $\tau$ on $H_{\infty}$ with parameter $z$ such that $\tau\left(T_{0}^{\prime} T_{1}^{\prime} \cdots T_{k-1}^{\prime}\right)=y_{k}$ for all $k \geq 1$. We restrict this Markov trace to $H_{\infty}^{\prime}$ and it remains to check that the value of $\tau$ on $U_{1}^{\prime} \cdots U_{2 k-1}^{\prime}$ indeed equals $y_{2 k}$. This is done as follows. We rewrite the elements 
$U_{i}^{\prime}$ in terms of the generators of $H_{\infty}$ and obtain that

$$
U_{i}^{\prime}=g_{i} \cdots g_{2} T g_{1} T g_{1}^{-1} g_{2}^{-1} \cdots g_{i}^{-1}=T T_{i}^{\prime} \quad \text { for all } i \geq 1 .
$$

Hence we must compute the value of $\tau$ on $\left(T T_{1}^{\prime}\right) \cdots\left(T T_{2 k-1}^{\prime}\right)$. While, on the level of $W_{\infty}$, the elements $t$ and $t_{i}$ commute with each other, this is no longer true on the level of $H_{\infty}$ for the elements $T$ and $T_{i}^{\prime}$. By [GL], Lemma 2.4(c), we have the commutation rule $T T_{2 i-1}^{\prime}=T_{2 i-1,0}^{\prime} T$ where $T_{2 i-1,0}^{\prime}=g_{2 i-1} \cdots g_{2} g_{1}^{-1} T g_{1} g_{2}^{-1} \cdots g_{2 i-1}^{-1}$. We apply this rule to every factor with an odd index; this will produce, for each $i$, a factor $T T$ between the $(2 i-1)$-th and the $2 i$-th term. Since $Q=1$ we have $T T=1_{H}$, and hence our desired trace value equals

$$
\tau\left(T_{1,0}^{\prime} T_{2}^{\prime} \cdots T_{2 k-2}^{\prime} T_{2 k-1,0}^{\prime} T\right)=\tau\left(T_{0}^{\prime} T_{1,0}^{\prime} T_{2}^{\prime} \cdots T_{2 k-2}^{\prime} T_{2 k-1,0}^{\prime}\right)
$$

The argument on the right hand side now has a form to which we can apply the results [GL], (4.4), on the evaluation of Markov traces for type B. By [loc. cit.], we can replace each $T_{i, 0}^{\prime}$ by an arbitrary element of the form $x T x^{-1}$ with $x=g_{i}^{ \pm 1} \cdots g_{1}^{ \pm 1}$, without affecting the trace value. Applying this to the factors $T_{2 i-1,0}^{\prime}$ yields that the above value in fact equals

$$
\tau\left(T_{0}^{\prime} T_{1}^{\prime} \cdots T_{2 k-1}^{\prime}\right)=y_{2 k}
$$

This completes the proof.

Finally, we can establish the following improvement to Proposition 6.1.

Corollary 6.3. Let $\tau: H_{\infty}^{\prime} \rightarrow A$ be a Markov trace with parameter $z$. Let $n \geq 1$ and $d_{i}^{\prime} \in \mathcal{D}^{\prime}\left(D_{i}\right)$ for $1 \leq i \leq n$. Then

$$
\tau\left(d_{1}^{\prime} \cdots d_{n}^{\prime}\right)=z^{a} \tau\left(U_{1}^{\prime} \cdots U_{2 b-1}^{\prime}\right)
$$

where $a$ is the number of factors $d_{i}^{\prime}$ which are equal to $g_{i-1}$ or $U$, and $2 b-1$ is the smallest odd number less than or equal to the number of factors $d_{i}^{\prime}$ which are equal to $U_{i-1}^{\prime}$.

P r o of. We only sketch this. By Theorem 6.2, the trace $\tau$ is the restriction of a Markov trace on $H_{\infty}$, which we shall denote by the same symbol. We can rewrite each $U_{i}^{\prime}$ as $T T_{i}^{\prime}$ and obtain an expression for $d_{1}^{\prime} \cdots d_{n}^{\prime}$ in terms of the generators of $H_{n}$. If there is a factor $U$ we also rewrite it as $T g_{1} T$. Now we use the commutation rule for $T$ and $T_{i}^{\prime}$ already mentioned in the proof of Theorem 6.2 to cancel intermediate factors $T$. The resulting expression either has signed block form in $H_{n}$ or is conjugate to such an expression by $T$ (but possibly with factors $T_{i, 0}^{\prime}$ instead of $T_{i}^{\prime}$ ). Performing this conjugation if necessary (which does not effect the trace value!) and using once more the evaluation rule in [GL], (4.4), we conclude that the value of $\tau$ on $d_{1}^{\prime} \cdots d_{n}^{\prime}$ is equal to the value on an element of the form $d_{1}^{\prime \prime} \cdots d_{n}^{\prime \prime}$ where each $d_{i}^{\prime \prime}$ either equals $g_{i-1}$ or $T_{i-1}^{\prime}$, and the number of factors $T_{i-1}^{\prime}$ is even. Now Lemma 5.2 implies that this value equals $z^{a} \tau\left(T_{0}^{\prime} \cdots T_{2 b-1}^{\prime}\right)$ for some $a, b$. We can argue as above in the proof of Theorem 6.2 to compare the values of $\tau$ on products $T_{0}^{\prime} T_{1}^{\prime} \cdots$ with those on products $U_{1}^{\prime} U_{2}^{\prime} \cdots$, and hence get the desired conclusion.

\section{Concluding remarks and open questions}

7.1. Relations with knot theory. Iwahori-Hecke algebras of type $A$ can also be described as quotients of the group algebras of Artin's braid groups. The motivation for 
introducing Markov traces in type $A$ is then given by Markov's Theorem in classical knot theory (see [Jo], Section 4). Now braid groups exist for any given type of finite Coxeter groups (cf. $[\mathrm{BS}]$ ), and the corresponding Iwahori-Hecke algebras again are quotients of their group algebras. Does there exist a knot theory in some suitable topological space such that the Markov traces for type $B$ and $D$ yield invariants for isotopy classes of oriented knots and links in an analogous way as Ocneanu's trace leads to the Jones polynomial? S. Lambropoulou [La] has shown that this is indeed the case for type $B$. The same question for type $D$ seems to be open.

7.2. Relations with $R$-matrices. Turaev has given an interpretation in terms of $R$ matrices for the construction of generalized Markov traces on classical braid groups (see [Bi], Section 3). In this way, invariants like the Jones polynomial and the Kauffman polynomial can all be derived in a uniform way. Does there exist such an $R$-matrix interpretation for the Markov traces discussed in this paper? (I wish to thank J. Birman for mentioning this problem to me.) In type $B$ and in the special case where there exists some $y \in A$ such that $\tau\left(T_{0}^{\prime} T_{1}^{\prime} \cdots T_{k-1}^{\prime}\right)=y^{k}$ for all $k \geq 1$ (notation of (5.4)), such an interpretation has been given by T. tom Dieck in [Di], $\S 4$ and 6 . The question seems to be open for general choices of the $y_{k}$ 's, as well as for type $D$.

7.3. The problem of weights. Let $\mathbf{K H}$ be the "generic" Iwahori-Hecke algebra of type $A_{n-1}, B_{n}$ or $D_{n}$, defined over an algebraically closed field as in the proof of Theorem 4.2. Then every trace function on $\mathbf{K H}$ is a linear combination of the characters corresponding to the irreducible representations of $\mathbf{K H}$. (These irreducible characters are labelled by partitions or pairs of partitions in a similar way as this was the case for the conjugacy classes of the underlying Coxeter groups.) In particular, it must be possible to express the Markov traces discussed in this paper as linear combinations of the irreducible characters, where the coefficients are called "weights". For Ocneanu's original trace function in type $A$ these weights are known and given in [Jo], pp.345. The weights in type $B$ and $D$ do not seem to be known.

\section{References}

[Bi] J. Birman, New points of view in knot theory, Bull. Amer. Math. Soc. 28 (1993), 253287.

[Bo] N. Bourbaki, Groupes et algèbres de Lie, Chap. IV, V, VI, Hermann, Paris, 1968.

[BS] E. Brieskorn and K. Saito, Artin-Gruppen und Coxeter-Gruppen, Invent. Math. 17 (1972), 245-271.

[Ca] R.W. Carter, Conjugacy classes in the Weyl group, Compositio Math. 25 (1972), 1-59.

[CR] C.W. Curtis and I. Reiner, Methods of representation theory, Vol. 2, Wiley, 1987.

[Di] T. tom Dieck, Knotentheorien und Wurzelsysteme, Teil II, preprint, Math. Gottingensis 44, 1993.

[GL] M. Geck and S. Lambropoulou, Markov traces and knot invariants related to IwahoriHecke algebras of type B, J. reine angew. Math. 482 (1997), 191-213.

[GP] M. Geck and G. Pfeiffer, On the irreducible characters of Hecke algebras, Advances in Math. 102 (1993), 79-94. 
[GR] M. Geck and R. Rouquier, Centers and simple modules for Iwahori-Hecke algebras, in: Finite Reductive Groups: Related Structures and Representations, Progress in Math. 141, Birkhäuser, 1997, 251-272.

[Jo] V.F.R. Jones, Hecke algebra representations of braid groups and link polynomials, Annals Math. 126 (1987), 335-388.

[La] S. Lambropoulou, Solid torus links and Hecke algebras of B-type, in: Proceedings of the Conference on Quantum Topology, D.N. Yetter (ed.), World Scientific Press, 1994.

[Pf] G. Pfeiffer, Charakterwerte von Iwahori-Hecke-Algebren von klassischem Typ, Aachener Beiträge zur Mathematik 14, Verlag der Augustinus-Buchhandlung, Aachen, 1995. 